\title{
X-ray time lags from a pivoting power law in black holes
}

\author{
E. Körding ${ }^{1}$ and H. Falcke $e^{1,2,3}$ \\ 1 Max-Planck-Institut für Radioastronomie, Auf dem Hügel 69, 53121 Bonn, Germany \\ 2 Radio Observatory, ASTRON, Dwingeloo, PO Box 2, 7990 AA Dwingeloo, The Netherlands \\ ${ }^{3}$ Department of Astronomy, University of Nijmegen, PO Box 9010, 6500 GL Nijmegen, The Netherlands
}

Received 11 March 2003 / Accepted 13 October 2003

\begin{abstract}
Most black hole candidate X-ray binaries show Fourier time lags between softer and harder X-rays. The hard photons seem to arrive up to a few ms after the soft for a given Fourier frequency of the perturbation. The energy dependence of the time lags has a roughly logarithmic behavior. Up to now most theories fail to explain the observed magnitude and Fourier frequency dependence of the lags or fail other statistical tests. We show that the time lags can arise from a simple pivoting power law model, which creates the logarithmic dependence on the photon energy at once. A pivoting power law arises naturally from jet/synchrotron models for the X-ray emission, but may also be applicable to corona models. A hint to the coherence features of the light-curves can be obtained from the power spectral density, which can be decomposed into a few broad Lorentzians that could arise from a couple of strongly damped oscillators with low quality factors below one. Using small variations of the power law index for each Lorentzian separately the lags can be derived analytically. They show the correct Fourier frequency dependence of the time lags. If one assumes variations of the power law index by \pm 0.2 the model can account for the observed magnitude of the time lags in Cyg X-1. The model can also be applied to TeV blazars, where a pivoting power law and hard lags have been observed directly in some cases. As a further test we calculated the cross- and auto-correlation functions for our model, which also show qualitatively the observed behavior. The auto-correlation function for higher energies has a narrower peak than at lower energies and the cross-correlation function is asymmetric but peaks nearly at zero. The coherence function for the model is in agreement with the observed data in the Fourier regime, where the model is valid.
\end{abstract}

Key words. X-rays: binaries - accretion, accretion disks - black hole physics - radiation mechanisms: non-thermal

\section{Introduction}

The central part of active black holes seems to consist of the black hole with an accretion disk surrounded by a hot corona (see e.g., Sunyaev \& Trümper 1979; Haardt \& Maraschi 1991) and a jet (e.g., Spencer 1979; Mirabel \& Rodríguez 1999; Fender 2001). However, up to now the accretion flow of black holes, jets, their connection, and their relative prominence are not well understood. The most common active black holes are active galactic nuclei (AGN) and black hole X-ray binaries (BHXRBs).

To constrain models and physical parameters of these objects it is important to access all observable quantities. Besides the spectra the variability is of high importance as it can reveal information about the central engine and its dynamics. Strong variability is a common phenomenom for XRBs (see e.g., van der Klis 1989). In BHXRBs the X-ray emission is commonly explained by an accretion disk and a Comptonizing corona (see, e.g. Shapiro et al. 1976; Sunyaev \& Trümper 1979; Haardt \& Maraschi 1991), but there may also be significant contributions from synchrotron emission from a jet (Markoff et al. 2001; Falcke \& Biermann 1999). The jet/synchrotron

Send offprint requests to: E. Körding, e-mail: koerding@mpifr-bonn.mpg.de model predicts a rigid power law that can only vary in amplitude and in spectral index. Variability in Comptonization models can lead to a power law X-ray spectrum as well (see e.g., Kylafis \& Klimis 1987). Here we will investigate whether the short term variability of active black holes can be explained with a rigid pivoting power law model. We will concentrate on BHXRBs as detailed light-curves are available, but applications to AGN are as well possible.

Usually BHXRBs appear in two distinct states: the hard state (low flux levels accompanied with a hard power law spectrum) and the soft-state (normally higher flux and a soft X-ray spectrum, see e.g., van der Klis 1994). In the hard-state a relativistic jet can usually been seen in radio observations (e.g., Fender 2001). We will focus our studies on the hard state, where the X-ray spectrum is dominated by a power law.

A BHXRB in the hard state shows significant short time $(0.1-100 \mathrm{~Hz})$ variability with a root mean square $(\mathrm{rms})$ around 20\% (see e.g., van der Klis 1995). It is therefore possible to make a detailed statistical analysis of the observed light curves. The light curves at different photon energies are well correlated as the cross-correlation function peaks nearly at unity. Furthermore, the coherence function (Vaughan \& Nowak 1997 ) is nearly unity for a wide range of Fourier frequencies. However, one often observes hard lags, e.g. the hard photons 
lag behind the soft photons up to a few milliseconds (e.g., see Nolan et al. 1981; Miyamoto \& Kitamoto 1989; Miyamoto et al. 1991; Pottschmidt et al. 2000, for a definition of phase lags see below, Eq. (13)). The existence of hard lags has been explained using Comptonization models. Soft photons will be repeatedly up-scattered in a large corona, as the harder photons need more inverse Compton processes to reach their energy this results in hard lags. For studies using coronae see e.g., Miyamoto et al. (1991), Nowak et al. (1999), Malzac \& Jourdain (2000), Poutanen (2002), or Böttcher et al. (2003). As already noted by these authors, this explanation has the problem that one needs huge coronae and the Fourier frequency dependence of the X-ray time lags cannot be reproduced (but see also Poutanen \& Fabian 1999).

Additionally the observed auto-correlation is not reproduced well (see e.g., Maccarone et al. 2000). A different approach has been made by Kotov et al. (2001), where the authors explain the phase lags with the response of the accretion disk to perturbations and present a short discussion of the effects of a pivoting power law.

By the term pivoting power law we mean that the X-ray spectrum at different times can always be described by a power law, which only varies in the power law index and the overall intensity. We mostly consider the case where the amplitude and the power law index are correlated.

The idea of a pivoting power law model arises from recent theoretical and observational results. The spectrum of BHXRBs can be well described using a coupled jet/accretion disk model (see Markoff et al. 2001). Here the disk (possibly an optically thin accretion disk, e.g., such as ADAFs and related solutions, Narayan \& Yi 1995, plus a standard disk) is only visible as an additional component in the UV, while the flat spectrum at radio and optical wavelength and the power law in the X-rays is created by synchrotron and inverse Compton emission from the jet. In particular, the hard X-ray power law is explained as optically thin synchrotron emission from a single region at a few hundred Schwarzschild radii from the black hole. The power law index depends on plasma parameters (e.g., electron temperature, adiabatic index), and may therefore respond to changes of the jet power and the accretion rate. As the total intensity depends on these parameters as well, the flux and the power law index should be correlated. The jet/synchrotron model therefore suggests that the X-ray emission behaves like a pivoting power law.

Within the jet/disk picture of Markoff et al. (2001), TeV Blazars like Mrk 421 or Fanaroff-Riley class I radio galaxies (Fanaroff \& Riley 1974, the unbeamed parent population of BL Lacs within the unified scheme, Urry \& Padovani 1995) show many features of BHXRBs in the low/hard state, namely a domination of the spectral energy distribution by jet emission. The connection of XRBs in the hard state and jet dominated AGN is discussed in Falcke et al. (2003). Mrk 421, for example, shows hard lags and a positive hardness/flux correlation (Zhang 2002). The hardness seems to show a hysteresis effect, e.g. the power law index seems to respond slightly after the variation of the total intensity. If BHXRBs also have a power law from their jets, a similar pivoting power law could play an important role. Hard lags and positive or negative hardness-flux correlations have also been found in Seyferts and other AGN see e.g., Chiang et al. (2000) or Lamer et al. (2003).

A pivoting power law may also be applicable for Comptonization models. Analyzing long term variability (timescales of days) of BHXRBs Zdziarski et al. (2003) suggest the existence of a pivoting power law with a pivot point around $50 \mathrm{keV}$ and explains the behavior using Comptonization in a corona. They find a negative correlation between flux and hardness. These long term variations arise probably from a different source of variability (e.g., the accretion rate or an other unknown parameter, see Homan et al. 2001) than the short term variations studied here (maybe created by magnetohydrodynamic instabilities, see Psaltis \& Norman 2002, or other unknown sources). Thus, it is yet unclear if such a correlation holds for fast variations and the true hard state.

In this paper we will analyze in a general way the effects of a pivoting power law model, where the power law index is correlated with the flux. We calculate the effect on the phase lags and the auto- and cross-correlation functions, and present a Monte Carlo simulation of the coherence function. In addition to the work by Kotov et al. (2001), who also discussed the possibility that the power law index is directly correlated with the flux, we include a response time for the change of the power law index as a function of intensity.

In Sect. 2 we describe our parameterization and model. With these definitions we derive a general analytic solution for phase lags and cross-correlation functions for a pivoting power law in Sect. 3. In Sect. 4 the analytic result is compared with a Monte Carlo simulation. In the last two sections we discuss our model in the context of data from Cygnus X-1 and present our conclusions.

\section{Parameterization of the pivoting power law model}

As we try to calculate the time lags with an analytical approximation it is important to parameterize our pivoting power law model around a reference photon energy $\epsilon_{0}$ near the observed energies. Let the flux $S$ of our source be a function of photon energy $\epsilon$ and time $t$

$S(\epsilon, t)=A(t)\left(\frac{\epsilon}{\epsilon_{0}}\right)^{-\alpha+\beta(t)}$,

where $\alpha$ represents the constant part of the spectral index while $\beta(t)$ accounts for the variations. The function $A(t)$ describes the flux at the reference energy $\epsilon_{0}$. As we will consider the case that $A(t)$ and $\beta(t)$ are correlated, the reference energy $\epsilon_{0}$ will not be the pivot point defined by the minimum of the rms.

If the changes in spectral index are small and we are observing photon energies near the reference energy $\left(\ln \left(\frac{\epsilon}{\epsilon_{0}}\right) \beta(t) \ll 1\right)$ we can expand the equation

$S(\epsilon, t)=A(t)\left(\frac{\epsilon}{\epsilon_{0}}\right)^{-\alpha}\left(1+\beta(t) \ln \left(\frac{\epsilon}{\epsilon_{0}}\right)\right)$,

and find in Fourier space, denoted by $\hat{S}$ :

$\hat{S}(\epsilon, \omega)=\left(\frac{\epsilon}{\epsilon_{0}}\right)^{-\alpha}\left(\hat{A}(\omega)+\widehat{\beta A}(\omega) \ln \left(\frac{\epsilon}{\epsilon_{0}}\right)\right)$. 
As we are interested in phase lags, depending on the coherence features of $A(t)$, it is inappropriate to use red noise for the light curve. Information on the coherence of the light curve can be guessed from the power spectral density (PSD) defined as $\operatorname{PSD}(\omega)=\hat{A}^{*}(\omega) \hat{A}(\omega)$, where the star denotes complex conjugation. We note that the PSD of many BHXRBs can be well described by a sum of a few broad Lorentzians

$\operatorname{PSD}(\omega)=\sum P_{\omega_{i}, R_{i}, Q_{i}}(\omega)$,

with

$P_{\omega_{i}, R_{i}, Q_{i}}(\omega)=\frac{4 R_{i}^{2} Q_{i} \omega_{i}}{\omega_{i}^{2}+4 Q_{i}^{2}\left(\omega-\omega_{i}\right)^{2}}$,

where one Lorentzian can be centered around $\omega=0$ (see Nowak 2000; Pottschmidt et al. 2003; Belloni et al. 2002). This definition of a Lorentzian follows Belloni et al. (2002). The quality factor $Q$ is a measure of the full width half maximum (FWHM) $Q=\frac{\omega}{2 \pi \mathrm{FWHM}}$. The normalization factor $R$ describes the amplitude of the Lorentzian. It is connected to the total rms amplitude as

$\mathrm{rms}_{i}=R_{i} \sqrt{\frac{1}{2}-\frac{\tan ^{-1}\left(-2 Q_{i}\right)}{\pi}}$

(see e.g., Pottschmidt et al. 2003). Lorentzians usually arise from damped oscillating systems, for example they are used to describe the spectral shape of a laser. The quality factor $Q$ describes how strong the oscillator is damped, a high $Q$ denotes a nearly undamped system with a strongly peaked PSD, while a low value for $Q$ yields a highly damped system with an asymmetric, weakly peaked PSD. Usually around four Lorentzians with a quality factors $Q \lesssim 1$ are needed to fit the PSD of XRBs in the low/hard state. As the origin of these broad Lorentzians is still unknown we assume that each Lorentzian is created by a strongly damped oscillator excited at random times. For example, these oscillators could be due to excitations at different locations on the accretion disk with a Fourier frequency defined by the Keplerian rotation that may or may not be transfered into the jet. Other possible explanations include magnetohydrodynamic instabilities (Psaltis \& Norman 2002) or jet precession.

To simplify the discussion we first look at only one broad Lorentzian centered around $\omega_{0}$. We assume that the variability is created by a damped oscillator. To generate our light-curve we use a simple shot noise model (for shot noise models see e.g., Terrell 1972; Lochner et al. 1991; Negoro et al. 2001). Let us first assume that the light curve of the BHXRB can be described as $F(t)$, if this oscillator has only been excited at $t=0$ with a unit excitation. The overall light curve will be a superposition of many excitations at random times and amplitudes. If $\lambda_{i}$ describes the amplitude of the excitation at the time $t_{i}$ we can write

$A(t)=A_{\mathrm{DC}}+\sum_{i} \lambda_{i} F\left(t-t_{i}\right)$,

where $A_{\mathrm{DC}}$ describes the constant offset of the flux. The amplitudes $\lambda_{i}$ and the excitation times $t_{i}$ are random variables. We choose the normalization of $F(t)$ such that $\left\langle\lambda^{2}\right\rangle=1$. Using this process we create a light curve that has the observed PSD and the coherence properties given by the oscillator.

It is unclear whether such simple shot noise models can describe the light-curves of XRBs (see e.g., Lochner et al. 1991). However, we have to disentangle the contributions of the different broad Lorentzians to apply our model, so it is very hard to test a pivoting power law model using observed light curves. Shot noise is therefore one of the best possibilities to create artificial light curves available. Furthermore, this approach enables us to give an analytic solution for the phase lags and the cross-correlation function. We discuss below the effect of different coherence properties. The main result is likely to be independent of the shot noise assumption.

Transfered into Fourier-space we find

$A(\omega)=A_{\mathrm{DC}} \delta(\omega)+\sum_{i} \lambda_{i} \hat{F}(\omega) \mathrm{e}^{\mathrm{i} \omega t_{i}}$.

The PSD of a complex damped oscillator is a Lorentzian, but as we are interested in real solutions for the light curve we have to use a linear combination of the real and the imaginary part of the damped complex oscillator. The two fundamental real solutions are the instantaneously excited oscillator (cosine)

$\hat{F}_{c}(\omega)=\frac{1}{2}\left(\hat{H}_{+}+\hat{H}_{-}\right)$

and

$\hat{F}_{s}(\omega)=\frac{1}{2 \mathrm{i}}\left(\hat{H}_{+}-\hat{H}_{-}\right)$

the sine combination, where $\hat{H}_{ \pm}=\frac{2 R \sqrt{Q \omega_{0}}}{\omega_{0}-2 \mathrm{i} Q\left(\omega \pm \omega_{0}\right)}$ is the Fourier transform of the complex oscillator with frequency $\omega= \pm \omega_{0}$. We note that the spectral form of the cosine combination $F_{c}$ declines with $\omega^{-2}$ like the Lorentzian, while the sine term drops with $\omega^{-4}$.

Given the light curve $A(t)$ we have to choose a physical response of the power law index $\beta(t)$. Whatever model one uses for the X-ray emission (Comptonization or jet model) the spectral index depends on physical properties near the accreting object. It is therefore likely that $\beta(t)$ will respond to changes of the accretion rate. As the region of emission has a characteristic size, $\beta$ may not follow $A(t)$ directly, but may respond a bit later. It is also possible that the emission mechanism has a response time itself (e.g., for the jet model how fast is the particle acceleration mechanism responding). We will take this into account by introducing a response time $\tau$. The first order approximation of $\beta$ will therefore depend linearly on $A(t-\tau)$. We assume that $\tau$ will be a small fraction $(a)$ of the period of the center frequency of the Lorentzian, i.e. $\tau=a 2 \pi / \omega_{0}$ with $0 \leq a<1$.

The first order approximation form of $\beta$ and the simplest form with the described properties is

$\beta(t)=\gamma A_{\mathrm{AC}}(t-\tau)$,

where the subscript AC marks the contributions to the light curve from the damped oscillators. The constant part of a variable will be marked with a subscript DC throughout this paper. In the case of a positive hardness/flux correlation, as seen in Mrk 421 and other Blazars, the parameter $\gamma$ is positive. For a negative correlation, seen in long timescale variablity of some 
BHXRBs, one has to use $\gamma<0$. For illustrative purposes we first use a positive hardness/flux correlation $(\gamma>0)$ in our calculations and discuss the other case in a separate subsection.

In Fourier representation one finds for $\beta$

$\hat{\beta}(\omega)=\gamma \mathrm{e}^{\mathrm{i} \omega \tau} \hat{A}_{\mathrm{AC}}(\omega) \hat{D}(\omega)$,

where we included an additional damping factor $D(\omega)$. It should take into account the damping of the system creating the power law, i.e. if $\omega$ is too big, the oscillations are so fast that they average out, and the power law index $-\alpha+\beta(t)$ does not change anymore.

\section{Analytic results}

\subsection{Definition and energy dependence of phase lags}

The phase lag measures the phase change (corresponding to a time delay) between the light curves at two photon energies in phase space. The Fourier phase lag $\phi(\omega)$ is defined as the argument of the average cross power spectrum

$\phi(\omega)=\arg \left\langle\hat{S}\left(\epsilon_{1}, \omega\right)^{*} \hat{S}\left(\epsilon_{2}, \omega\right)\right\rangle$

see e.g., Nowak et al. (1999). The Fourier phase lag can be translated to time lags by dividing through $\omega$. The time lags have a simple interpretation in the time domain, as they measure the time difference between an outburst at two different photon energies at a given Fourier frequency. To calculate the phase lag from a light-curve we start from

$\sin \phi=\frac{\mathfrak{J}\left[\hat{S}^{*}\left(\epsilon_{1}, \omega\right) \hat{S}\left(\epsilon_{2}, \omega\right)\right]}{\left|\hat{S}\left(\epsilon_{1}, \omega\right)\right|\left|\hat{S}\left(\epsilon_{2}, \omega\right)\right|}$.

Using the linear approximation (Eq. (8)) the phase lag can be evaluated analytically (see also Kotov et al. 2001):

$$
\begin{aligned}
\sin \phi & \approx \frac{\mathfrak{J}\left[\left[\hat{A}^{*}+(\widehat{\beta A})^{*} \ln \left(\frac{\epsilon_{1}}{\epsilon_{0}}\right)\right]\left[\hat{A}+(\widehat{\beta A}) \ln \left(\frac{\epsilon_{2}}{\epsilon_{0}}\right)\right]\right]}{\hat{A}^{*} \hat{A}} \\
& \approx \frac{\mathfrak{J}\left[\hat{A}^{*}(\widehat{\beta A})\right]}{\hat{A}^{*} \hat{A}} \ln \frac{\epsilon_{2}}{\epsilon_{1}} .
\end{aligned}
$$

So, if $\hat{A}^{*}(\widehat{\beta A})$ has a nonzero imaginary part, the phase lag will vary with $\ln \epsilon$ as seen in the observations of BHXRBs. The photon energy dependence of the phase lag is therefore independent of the response $\beta(t)$ of the power law index, but the overall magnitude and the pase lag dependence on Fourier frequency $\omega$ depends on the choice of $\beta$.

\subsection{Fourier frequency dependence of the phase lag}

To derive the phase lag we start with Eq. (15). In our linear approximation the only unknown component is the Fourier transform of $\beta A$. Using the convolution theorem and the Fourier transform of $\beta$ from Eq. (12) we get:

$$
\begin{aligned}
\widehat{\beta A}= & \gamma \int \mathrm{e}^{\mathrm{i} \omega^{\prime} \tau} \hat{A}_{\mathrm{AC}}\left(\omega^{\prime}\right) \hat{A}\left(\omega-\omega^{\prime}\right) \hat{D}\left(\omega^{\prime}\right) \mathrm{d} \omega^{\prime} \\
= & \gamma \int \mathrm{e}^{\mathrm{i} \omega^{\prime} \tau} \hat{A}_{\mathrm{AC}}\left(\omega^{\prime}\right) \hat{A}_{\mathrm{AC}}\left(\omega-\omega^{\prime}\right) \hat{D}\left(\omega^{\prime}\right) \mathrm{d} \omega^{\prime} \\
& +\gamma A_{\mathrm{DC}} \hat{A}_{\mathrm{AC}}(\omega) \hat{D}(\omega) \mathrm{e}^{\mathrm{i} \omega \tau} .
\end{aligned}
$$

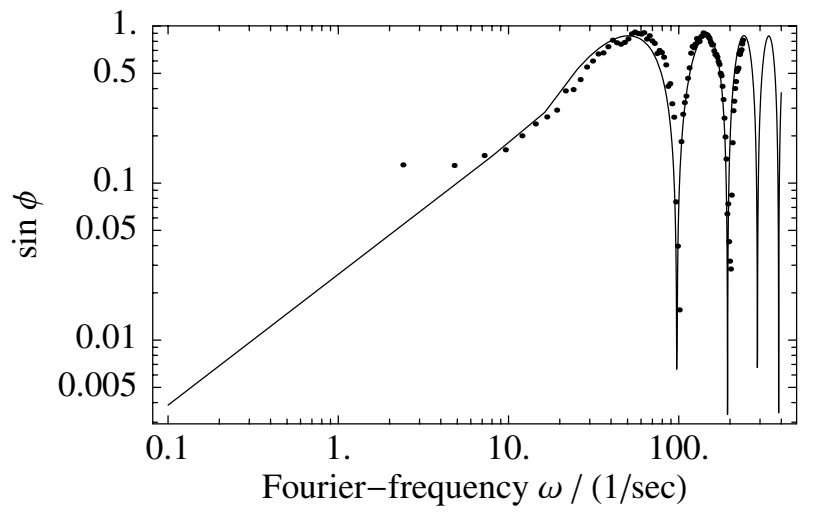

Fig. 1. Phase lag dependence on the Fourier frequency. The solid line represents the analytic approximation, the dots are the results of a Monte Carlo simulation. The deviation of the Monte Carlo simulation at lower frequencies is a numerical effect.

For the case $\hat{D}(\omega)=1$ it is possible to give an analytic calculation of the phase lag. The calculation is described in Appendix A and we just give the result for an instantaneously excited oscillator:

$\sin \phi=\gamma \ln \frac{\epsilon_{2}}{\epsilon_{1}}\left(A_{\mathrm{DC}} \sin (\tau \omega)+\frac{\left\langle\lambda^{3}\right\rangle}{\left\langle\lambda^{2}\right\rangle} \mathfrak{J}\left(\mathrm{e}^{\mathrm{i} \tau \omega} f_{1}(\omega)\right)\right.$,

where $f(\omega)$ is given in the appendix. The $\lambda$ denote the random excitation power of the oscillators. It is important to note that the calculations show that one can treat each excitation of the oscillator separately - the contributions of different excitations average out.

The first term in Eq. (17) is the result of the pivoting power law acting on the constant flux, while the second term represents the pivoting power law acting on the pulse itself. So the second term depends on $\frac{\left\langle\lambda^{3}\right\rangle}{\left\langle\lambda^{2}\right\rangle}$ and will therefore vanish if the $\lambda$ are distributed symmetrically around zero. In that case only the first term will contribute in that order of the perturbation series, but higher orders can be nonzero.

The behavior of the phase lag is illustrated in Fig. 1 (solid line). For small $\omega \tau$ the phase lags increase linearly with frequency. This means that the time lags are constant for small frequencies. Once $\omega \tau \approx \frac{\pi}{2}$, the phase lags start to oscillate. If $\tau$ is of the order of $\frac{2 \pi}{\omega_{0}}$ this will happen roughly at $\omega>\omega_{0}$.

The result that the phase lag starts to oscillate is at least partly due to our deterministic law of a fixed look-back time $\tau$. A more realistic approach would be that $\tau$ is itself a random variable, e.g., Gaussian distributed around a mean value. As the sine is linear for $\omega \tau \ll 1$ the phase lags for low Fourier frequencies will not change. However, for $\omega \tau \gtrsim 1$ the contributions to the phase lags for different $\tau$ will average out. The oscillation will be further reduced as the PSD of a real system is described by several Lorentzians, i.e. for $\omega \gg \omega_{0}$ a second Lorentzian will dominate the first one. It is therefore likely that one will never observe the oscillating part of the phase lag.

To take the probably statistical nature of $\tau$ and additional damping of the response of the power law into account, we choose a damping term $\hat{D}(\omega) \neq 1$, for example $\hat{D}(\omega)=\mathrm{e}^{-\delta\left(\frac{\omega}{\omega_{0}}\right)^{2}}$, which will cut off the phase lags at a given frequency. With an 


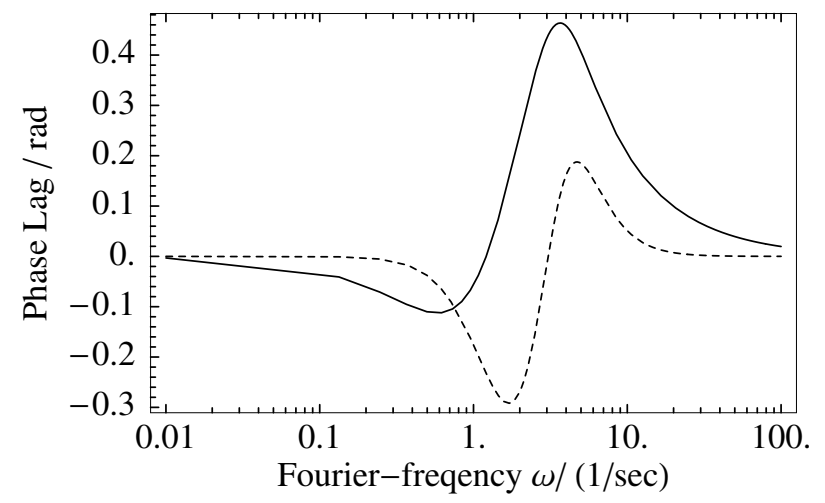

Fig. 2. Difference between the sine and cosine part. The magnitude of the sine lags (dashed) have been magnified by a factor of ten.

appropriately chosen damping the phase lag will not oscillate but stay at zero for higher Fourier frequencies.

Hence, we conclude that the phase lag in the case $\tau>0$ can be approximated by

$\sin \phi \sim \gamma \sin (\omega \tau) \hat{D}(\omega) \ln \frac{\epsilon_{2}}{\epsilon_{1}}$

and is independent of the exact shape of the pulses. We find hard lags for a positive hardness flux correlation and a positive look-back time. If one changes the parameters, e.g., negative hardness flux correlation and positive look-back times, soft lags can be obtained.

We can now verify that our simplification made in Eq. (7) was appropriate. We assumed that all excitations are identical and only vary in amplitude and excitation time. In a real system each excitation will have a different shape. But as the contributions to the phase lags from different excitations average out, each pulse contributes as if there are no other excitations (the sum in Eq. (A.4) only runs over the diagonal part). The overall phase lag will be the average of all pulses or the phase lag of an average shaped pulse. In the zeroth order approximation the coherence does not play a role for the phase lags (as long as there is a constant flux component). Thus the shot noise assumption is not a crucial ingredient for the model. The important assumption is only that the PSD can be decomposed into different Lorentzians, which do not interact with each other.

In the case that the power law index responds instantly to a change in the accretion rate, the first term of Eq. (17) will vanish and the phase lag depends to first order in $\gamma$ linearly on $\left\langle\lambda^{3}\right\rangle$. The lags would therefore vanish in the case of a symmetric distribution of the $\lambda$ (many systems show asymmetric excitations, for one example see Spruit \& Kanbach 2002). Furthermore, they depend strongly on the linear combination used for the pulse shape. The lags created by the sine and cosine term are shown in Fig. 2. Nevertheless, if the power law index $\Gamma$ is varying by $\Delta \Gamma \approx 0.2$, as will be used here later on, the phase lags due to the pivoting power law will contribute significantly to the observed phase lags. The sign of the lag changes with frequency as mentioned by Kotov et al. (2001), who evaluated an instantly changing power law for a real light curve.

\subsection{Cross-correlation function}

The phase lags depend only on the phase of the Fouriertransform and contain no information on the amplitude. To gain information also on the amplitudes we consider the auto-correlation and cross-correlation functions. The crosscorrelation function is defined as:

$$
\begin{aligned}
C\left(\epsilon_{1}, \epsilon_{2}, \bar{\tau}\right)= & \int\left(S\left(\epsilon_{1}, t\right)-\left\langle S\left(\epsilon_{1}\right)\right\rangle\right) \\
& \times\left(S\left(\epsilon_{2}, t+\bar{\tau}\right)-\left\langle S\left(\epsilon_{2}\right)\right\rangle\right) \mathrm{d} t .
\end{aligned}
$$

We have chosen the signs such that the cross-correlation function between a lower and a higher photon energy peaks to the right if we observe hard lags. Expressed in Fourier-space we find:

$C\left(\epsilon_{1}, \epsilon_{2}, \bar{\tau}\right)=\int \hat{S}_{\mathrm{AC}}\left(\epsilon_{1}, \omega\right)^{*} \hat{S}_{\mathrm{AC}}\left(\epsilon_{2}, \omega\right) \mathrm{e}^{-\mathrm{i} \omega \bar{\tau}} \mathrm{d} \omega$

If we insert the expanded expression (3) for $\hat{S}(\epsilon, \omega)$ and only consider terms up to $O\left(\gamma^{2}\right)$ we find:

$$
\begin{aligned}
C\left(\epsilon_{1}, \epsilon_{2}, \bar{\tau}\right)= & \int \hat{A}_{\mathrm{AC}}^{*} \hat{A}_{\mathrm{AC}} \mathrm{d} \omega \\
& +\int\left(\hat{A}^{*} \widehat{\beta A} \ln \epsilon_{2}+\widehat{\beta A}^{*} \hat{A} \ln \epsilon_{1}\right)_{\mathrm{AC}} \mathrm{e}^{-\mathrm{i} \omega \bar{\tau}} \mathrm{d} \omega .
\end{aligned}
$$

For simplicity we have set the reference energy $\epsilon_{0}=1$.

The first component of the integrand represents the autocorrelation function of the light curve at the reference energy:

$$
\int \hat{A}_{\mathrm{AC}}^{*} \hat{A}_{\mathrm{AC}} \mathrm{e}^{-\mathrm{i} \omega \bar{\tau}} \mathrm{d} \omega=\int \sum_{i} \lambda_{i}^{2} \hat{F}^{2}(\omega) \mathrm{e}^{-\mathrm{i} \omega \bar{\tau}}=: P(\bar{\tau}) .
$$

The second component takes a longer calculation using the results for $\widehat{\beta A}$ of Appendix A and is described in Appendix B. With the function $\mathcal{S}(\tau, \bar{\tau})$ defined in the Appendix we find for the cross-correlation function

$$
\begin{aligned}
C\left(\epsilon_{1}, \epsilon_{2}, \bar{\tau}\right)= & P(\bar{\tau})+\gamma\left(\ln \epsilon_{1} P(\bar{\tau}-\tau)+\ln \epsilon_{2} P(\bar{\tau}+\tau)\right. \\
& \left.+\ln \epsilon_{2} \mathcal{S}(\tau, \bar{\tau})+\ln \epsilon_{1} \mathcal{S}(\tau,-\bar{\tau})\right)
\end{aligned}
$$

As described in the Appendix $\mathcal{S}$ peaks at $\bar{\tau}=\tau$ and decays faster than $P(\bar{\tau})$.

Whether the auto-correlation function has a steeper peak for higher photon energies $\epsilon$ depends on the parameters. While the terms in the first line of Eq. (23) makes the auto-correlation function broader for higher energies $(\sim \log \epsilon)$ the terms in the second line have the opposite effect. For small $Q$ and $\tau$ the peak is steeper for higher photon energies while for larger values of $\tau$ and $Q$ the opposite effect is found.

If the excitations of the oscillator are symmetric, i.e. $\left\langle\lambda^{3}\right\rangle=0, \mathcal{S}$ vanishes, and only higher order terms contribute to the cross-correlation function. The feature that the crosscorrelation function can have a steeper component for higher energies remains in this case as seen in the Monte Carlo simulation.

A numerical evaluation of the cross correlation function with a damping factor of unity is shown in Fig. 3. The calculated auto-correlation function is, for the parameters used, 


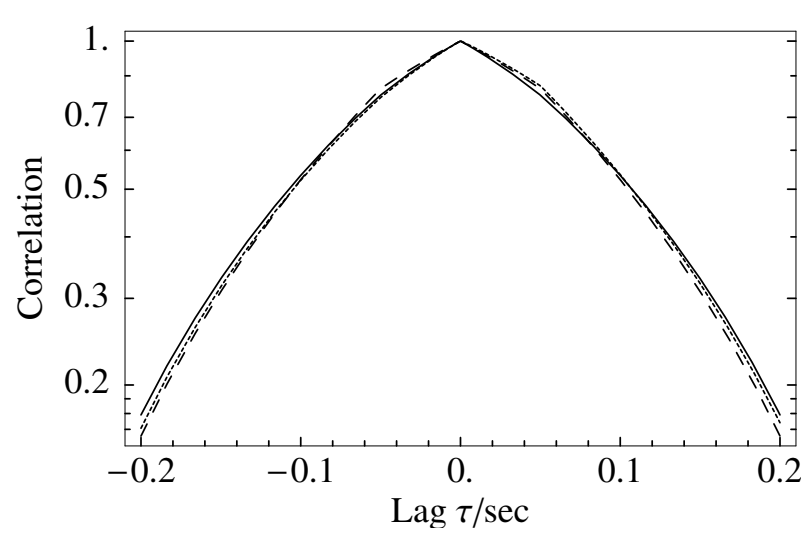

Fig. 3. Numerical evaluation of the calculated cross-correlation function for one Lorentzian only. solid line: low energy auto-correlation function. dashed: higher energy; dotted: cross-correlation function.

steeper for higher energy photons. The cross-correlation function between two energies lies in between and is slightly asymmetric. It is important to note that whether the auto-correlation function is steeper for higher photon energies depends strongly on the parameters, e.g. the look-back time $\tau$.

If one includes a damping factor $\hat{D}(\omega)$ that damps higher frequencies in the response of the power law index, the light curve itself will have less power in high Fourier frequencies for higher energies. Therefore the auto-correlation function will have a flatter peak for higher energies than without a damping factor. The effect described above (steeper auto-correlation function for higher energies due to the $\mathcal{S}$-term) and the effect of the damping factor can cancel each other partly.

\subsection{Negative correlation of hardness and flux}

In the previous sections we have considered a pivoting power law model with a positive hardness flux correlation $(\gamma>0)$ and a positive look-back time $\tau$. However Zdziarski et al. (2003) observed a negative hardness flux correlation and a pivot point around $50 \mathrm{keV}$ for Cyg X-1 in long term variablity (timescale of days). On shorter timescales Li et al. (1999) and Feng et al. (1999) report a negative correlation as well. Therefore a negative correlation can also be present on the short timescales discussed here. A negative hardness/flux correlation $(\gamma<0)$ and a positive look-back time ( $\tau>0$ ) leads to soft lags. Soft lags have for example been observed in X-ray burst oscillations (Ford 1999). However, the observed lags are approximately $1 \mathrm{rad}$, much larger than the lags discussed here. So this effect may be due to other mechanisms.

In order to create hard lags with a negative hardness/flux correlation $(\gamma<0)$ one has to use negative look-back times $\tau$. This means that the power law index is changed slightly before the flux changes. Now the pivot point defined by the rms is at higher photon energies as the reference frequency $\epsilon_{0}$. If $\epsilon_{0} \approx 3 \mathrm{keV}$ pivot points around $50 \mathrm{keV}$ can be reached. In this case two signs are changed in Eq. (18) resulting in hard lags as in the case of $\gamma>0$. If one changes the sign of $\tau$ we have to consider that the analytic behavior of Eq. (16) changes as well. The first term of the phase lag $(\sin \phi \sim \gamma \sin (\omega \tau))$ is the

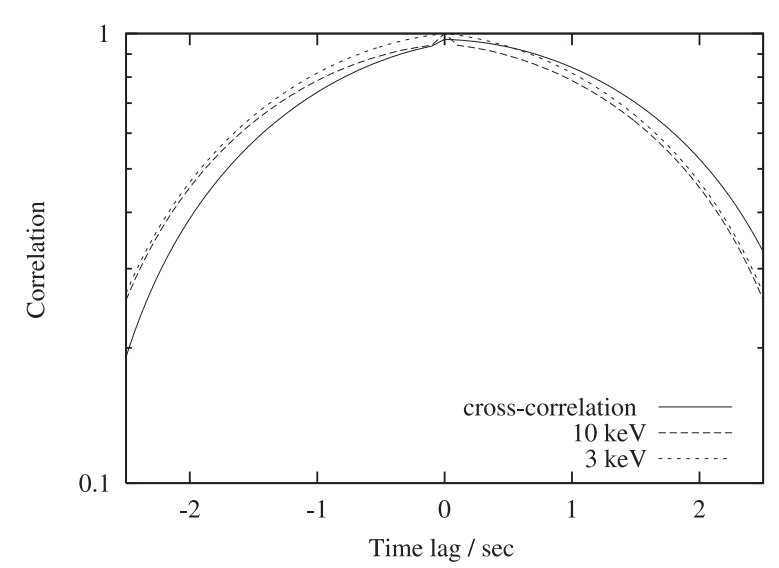

Fig. 4. Monte Carlo simulation of the calculated cross-correlation function for a negative hardness/flux correlation and a negative lookback time.

same for negative and positive correlation. However, the second term does not depend on $\mathfrak{J} \mathrm{e}^{\mathrm{i} \omega \tau}$ as before (Eq. (A.9), see Appendix A). We find instead

$\sin \phi=\gamma \ln \frac{\epsilon_{2}}{\epsilon_{1}}\left(A_{\mathrm{DC}} \sin (\tau \omega)+\frac{\left\langle\lambda^{3}\right\rangle}{\left\langle\lambda^{2}\right\rangle} \mathfrak{J}\left(f_{2}(\omega)\right)\right.$,

where $f_{2}$ is a rational function in $\omega$. If the constant flux $A_{\mathrm{DC}}$ dominates the overall flux the non oscillating second term will only be a minor correction. The phase lag from a pivoting power law with negative look-back times $\tau$ and negative correlation will therefore yield similar phase lags as before.

Even though the second term of the phase lags has a different structure, the auto-correlation function has the same analytic structure as in the case $\tau>0$ (Eq. (23)). Only the function $\mathcal{S}$ has to be changed. As $\gamma$ is now negative, the first line of Eq. (23) already yields a steeper auto-correlation function for higher photon energies $\epsilon$. As the second line depends on $\gamma$ as well, it can broaden the auto-correlation function. Which one of these effects dominates depends on the parameters used. Compared to the case with a positive hardness flux correlation, the auto-correlation function has a steeper decline for small values of $\bar{\tau}$ (see Fig. 4) and a slower one for larger $\bar{\tau}$.

In summary, the two cases positive hardness/flux correlation and positive look-back time and the case with a negative hardness/flux correlation and a negative look-back time have similar hard phase lags. Only the parameters have to be adjusted somewhat differently. However, while in the first case the auto-correlation function is flatter for small lags and steepens for larger lags, the opposite effect happens in the other case. With appropriately chosen parameters both possibilities seem to be able to reproduce the qualitative behavior seen in the observations of Cyg X-1 by Maccarone et al. (2000).

\section{Monte Carlo simulations}

The analytic results have used a linear approximation for the response of the power law. For observations near the reference photon energy $\frac{\epsilon}{\epsilon_{o}} \lesssim 1$ and small responses of the power law index $\gamma \ll 1$ this approximation will be valid. However, as one 
often observes photon energies with $\frac{\epsilon_{\text {high }}}{\epsilon_{\text {low }}} \gtrsim 10$ we created a Monte Carlo simulation. This will also enable us to consider a system with more than one broad Lorentzian. The light curve for one Lorentzian was generated as described by Eq. (7). The random variables $\lambda_{i}$ are chosen to be the absolute value of a Gaussian distribution and the excitation times $t_{i}$ are uniformly distributed. Each individual pulse is described by the sum of the cosine and sine damped oscillator $\left(F=F_{\mathrm{c}}+F_{\mathrm{s}}\right)$. Once the light curve at the reference photon energy is created we can calculate light curves at other photon energies using Eq. (1) and derive the phase lags. The comparison between the numerical results and the analytic calculation is shown in Fig. 1. We find that the analytic first order approximation and the Monte Carlo simulation are in good agreement.

If one assumes that the different oscillators creating the broad Lorentzians do not interact with each other one can calculate a light curve for a system described by multiple Lorentzians by creating light curves for each Lorentzian separately and then superpose these light curves to get the overall light curve. This is used in the application to Cyg X-1, in Sect. 5 .

Besides the phase lags we can calculate the cross- and autocorrelation functions of the light curve. The Monte Carlo simulation reproduces the effect seen in the analytic calculations: for the parameters used the auto-correlation function is steeper for higher energy photons than for the low energetic once. In Fig. 4 we show the result of the Monte Carlo simulation with a negative look-back time. The cross-correlation function is asymmetric as expected for hard lags.

\section{Applications to BHXRBs}

We are now able to apply the model to the BHXRB Cygnus X-1. Throughout this section we use data for Cyg X-1 which was taken from Nowak et al. (1999) and Kotov et al. (2001) for the photon energy dependence of the phase lag.

\subsection{Photon energy dependence of the phase lags}

The pivoting power law model predicts that the energy dependence of the phase lags is logarithmic (Eq. (15)). In Fig. 5 we show the phase lags measured with a constant Fourier frequency of $2.5 \mathrm{~Hz}$ (dots). The solid line represents the pivoting power law model. The reference energy $\epsilon_{0}$ used for the fit is $2.0 \mathrm{keV}$. For lower photon energies the accretion disk will become increasingly important and will start to dominate over the power law component. In this regime a simple pivoting power law will not be sufficient to describe the behavior and deviations from the logarithmic energy dependence of the lags are likely.

\subsection{Fourier frequency dependence of the phase lags}

The PSD of Cyg X-1 can be well fitted with four broad Lorentzians (see e.g., Nowak 2000; Belloni et al. 2002; Pottschmidt et al. 2003). The phase lag from each Lorentzian will dominate the overall phase lag around its center frequency. The idea is illustrated in Fig. 6 where we calculated

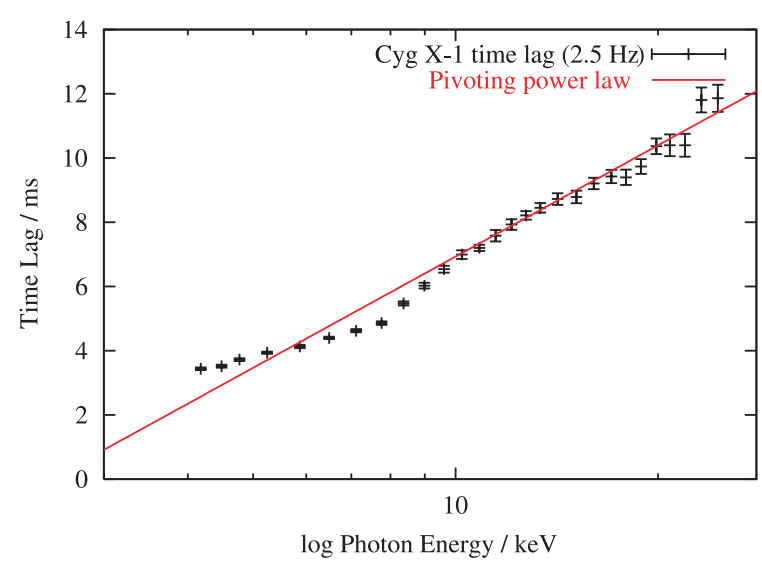

Fig. 5. Time Lags versus photon energy at a Fourier frequency of $2.5 \mathrm{~Hz}$. The solid curve represents the analytic solution for the pivoting power law model with a reference energy of $2.0 \mathrm{keV}$.

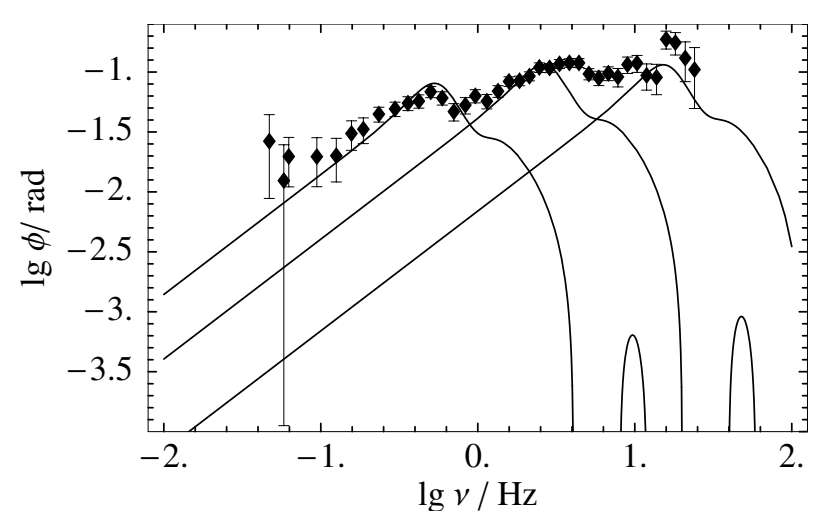

Fig. 6. Illustration for the superposition of the different broad Lorentzians. In the superposition every Lorentzian should dominate the phase lag around its center frequency.

the phase lags for each Lorentzian separately. The look-back time $\tau$ is always the same fraction of the center frequency of the Lorentzian, which reduces the number of free parameters. In this plot we assumed that the excitations are similar to those of an instantaneously excited oscillator and used as damping factor a Lorentzian with $Q=0.25$ (Center frequencies $f=0.25,1.2,7.3 \mathrm{~Hz}, \tau=0.03 f^{-1}$ ).

If we assume that the different broad Lorentzians do not interact with each other we can do the superposition with our Monte Carlo simulation. First we generate a separate light curve for each Lorentzian. In this way we have the possibility to consider that each broad Lorentzian has a different lookback time $\tau$, which we assumed to be a fixed fraction of the period of that Lorentzian. The overall light curve is the superposition of the four light curves. The PSD of the artificial light curve is shown in Fig. 7. The parameters of the Lorentzians are given in Table 1. The pulse of the shot noise is a superposition of the damped sine and cosine oscillator, so its PSD is not exactly a Lorentzian, but this only leads to small deviations of the overall PSD. The PSD has been used to find the overall normalization of the shot noise.

The damping factor $D(\omega)$ used in the Monte Carlo simulation is a Lorentzian centered around the center-frequency of oscillator creating the broad Lorentzians with the half quality 


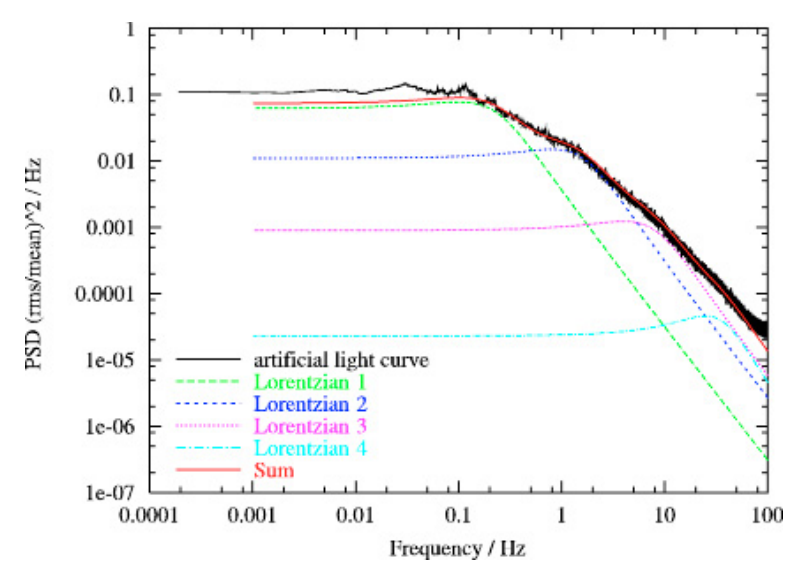

Fig. 7. The PSD of the artificial light curve together with the four broad Lorentzians. For their parameters see Table 1.

Table 1. The parameters used for Cyg X-1.

\begin{tabular}{lcccc}
\hline \hline Lorentzian & 1 & 2 & 3 & 4 \\
\hline$f_{\mathrm{c}}$ in $\mathrm{Hz}$ & 0.1 & 0.8 & 4.0 & 25.0 \\
$Q$ & 0.25 & 0.3 & 0.3 & 0.5 \\
$R$ & 0.22 & 0.25 & 0.16 & 0.06 \\
$f_{\text {peak }}$ in $\mathrm{Hz}$ & 0.22 & 1.55 & 7.7 & 35.0 \\
\hline
\end{tabular}

factor as the oscillator itself. The constant power law index $\alpha$ has been fixed to 1.7 , and the correlation factor $\gamma=0.26$. The look-back time was chosen to be $\tau=0.04 f^{-1}$, where $f$ denotes the center frequency of the Lorentzian. In Fig. 8 the phase lags from this Monte Carlo simulation are plotted in comparison with the observed values from Nowak et al. (1999). The parameters are summarized in Table 1 . The peak frequencies, where the Lorentzians contribute mostly to the rms, are also given in this table. The peak frequency $\left(f_{\text {peak }}=f \sqrt{1 /\left(4 Q^{2}\right)+1}\right)$ is often used for frequency correlations (see e.g., Nowak 2000). The parameters of the Lorentzians are within the range of the values given by Pottschmidt et al. (2003). The effect of the fourth Lorentzian on the phase lags is rather small, as this Lorentzian does not dominate the overall PSD at its center frequency.

The used parameters are not unique. For example, a small change of the hardness/flux correlation parameter $\gamma$ can be compensated by a change of the look-back time $\tau$. Furthermore, the central frequencies of the Lorentzians needed to fit the phase lags depend on the damping factors and the positions of the other Lorentzians. These parameters where chosen to give a steeper auto-correlation for higher photon energies (see below). If one would choose different fractions of the center frequency of the Lorentzian for the look-back time $\tau$ and allows for a different hardness/ flux correlation factor $\gamma$ for each Lorentzian better fits would be possible.

A similar result for the phase lags can be obtained using a negative hardness flux correlation and a negative lookback time. One possibility would be to choose $\gamma=-0.16$ and $\tau=-0.06 f^{-1}$ and to center the Lorentzians given in Table 1 at slightly different positions ( $f=0.15,1.2,6.0,25 \mathrm{~Hz}$ ).

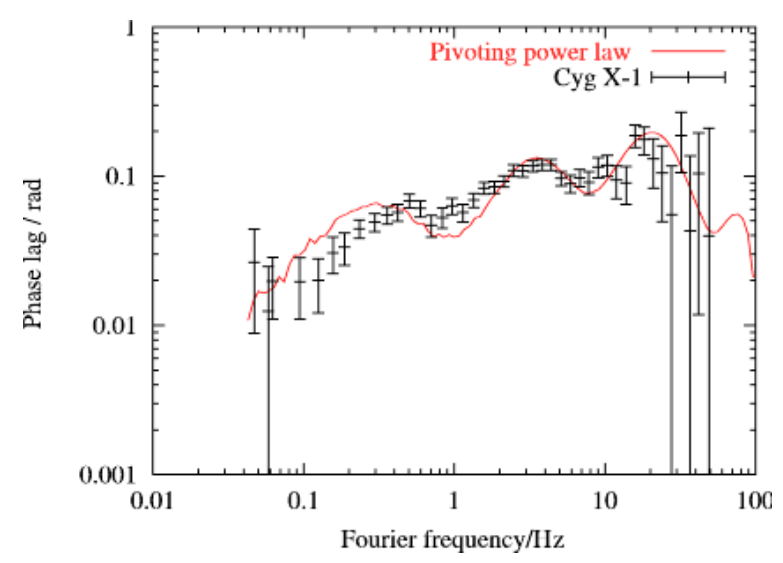

Fig. 8. Phase-lag dependence $(0-3.9 \mathrm{keV}$ to $8.2-14.1 \mathrm{keV})$ on frequency. Four Lorentzians with parameters given in Table 1 have been superposed with our Monte Carlo Code. The data were taken from Nowak et al. (1999).

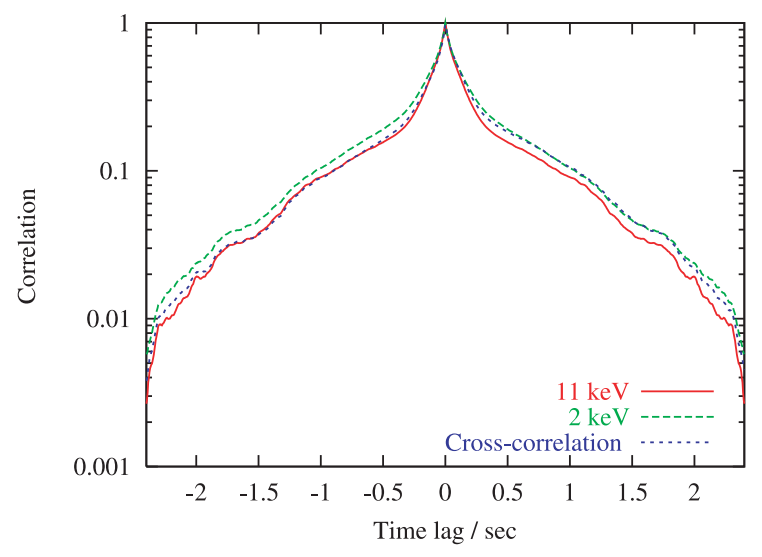

Fig. 9. Auto- and cross-correlation function created by the Monte Carlo simulation. See Table 1 for the used parameters.

\subsection{Auto- and cross-correlation function}

In addition to the phase lags we evaluated the auto- and crosscorrelation function for the simulated light curves at different photon energies. In Fig. 9 we show the auto- and crosscorrelation function for the parameters used in Fig. 8, here with a positive hardness/flux correlation and a positive lookback time. With these parameters the auto-correlation function is steeper for higher photon energies and the cross-correlation is slightly asymmetric. The plot shows qualitatively the same behavior as the observed correlation shown in Maccarone et al. (2000). However, this behavior depends strongly on the parameters, such as the look-back time $\tau$ or even the relative prominence of the different Lorentzians.

\subsection{Coherence function}

The coherence function measures the linear correlation of the two light curves at different photon energies. For an introduction and a discussion of its properties see Vaughan \& Nowak (1997). It is defined as

$\gamma_{I}^{2}(\omega)=\frac{\left|\left\langle\hat{S}^{*}\left(\epsilon_{1}, \omega\right) \hat{S}\left(\epsilon_{2}, \omega\right)\right\rangle_{\mathrm{d}}\right|^{2}}{\left\langle\left|\hat{S}\left(\epsilon_{1}, \omega\right)\right|^{2}\right\rangle_{\mathrm{d}}\left\langle\left|\hat{S}\left(\epsilon_{2}, \omega\right)\right|^{2}\right\rangle_{\mathrm{d}}}$ 


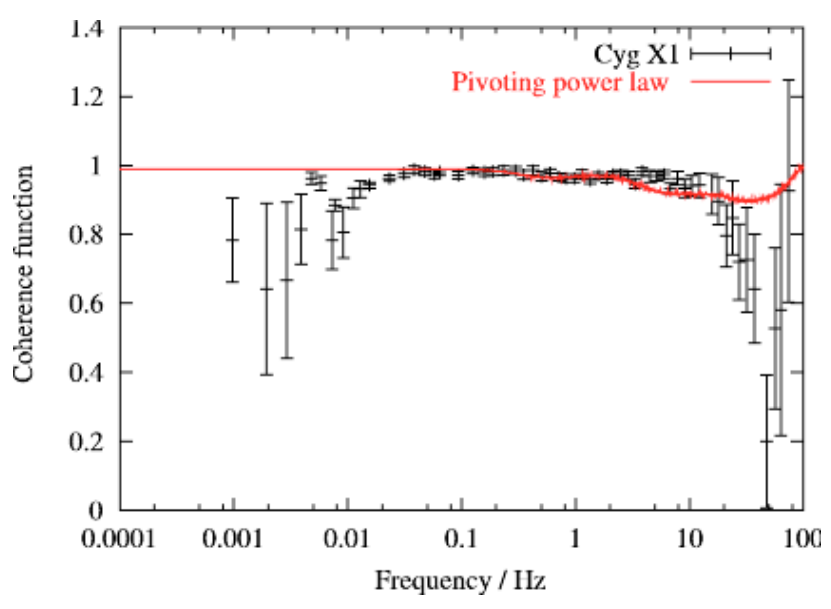

Fig. 10. Coherence function for a pivoting power law model. See Table 1 for the used parameters.

where $\langle.\rangle_{\mathrm{d}}$ denotes an average over different realizations of the statistical process, e.g. different light-curves. If the coherence function is unity the light-curves at different photon energies are connected by a linear transformation. To evaluate the coherence function for the pivoting power law model we simulated 100 different $5000 \mathrm{~s}$ light curves with a time resolution of $5 \mathrm{~ms}$. The result is shown in Fig. 10 together with the measured coherence function of Cyg X-1. The highest frequency Lorentzian included in our calculation is centered around $25 \mathrm{~Hz}$. Due to our damping factors the two light-curves are therefore perfectly correlated at higher Fourier frequencies. This leads to the rise of $\gamma_{I}^{2}(\omega)$ for higher Fourier frequencies to one. The rise is therefore an artifact of our choice of the damping factors and probably missing higher Lorentzians. In the low Fourier frequency domain $(\leq 0.01 \mathrm{~Hz})$ the PSD is usually dominated by a power law noise component (Nowak 2000), which may be responsible for the lower coherence function in this regime. We conclude that the model is consistent with the data in the Fourier frequency regime where the model is valid.

\subsection{Failed state transitions}

Pottschmidt et al. (2000 \& 2002) identified some flares of Cyg X-1 as "failed state transitions". During these flares the X-ray spectrum softens and that the rms amplitude decreases. The PSD is then usually dominated only by the second Lorentzian (peak frequency $\approx 3 \mathrm{~Hz}$ ) and to some extend the third Lorentzian (peak frequency $\approx 9 \mathrm{~Hz}$ ). The phase lags during the "failed state transitions" increases significantly in the $3-10 \mathrm{~Hz}$ regime, while it stays nearly constant compared to a normal hard state below $1 \mathrm{~Hz}$ and above $10 \mathrm{~Hz}$. The third and second Lorentzian dominate the PSD between 3 and $10 \mathrm{~Hz}$. This leads these authors to the conclusion that the increased phase lag can be attributed to these Lorentzians. It has already been pointed out by Nowak (2000) and Pottschmidt et al. (2003) that the phase lags could be reduced due to the superposition of the different Lorentzians. If one Lorentzian starts to dominate the overall PSD the phase lags will therefore increase. For example in Fig. 11 we reduced the strength of the first and third Lorentzian $(R=0.1)$ while leaving all other parameters

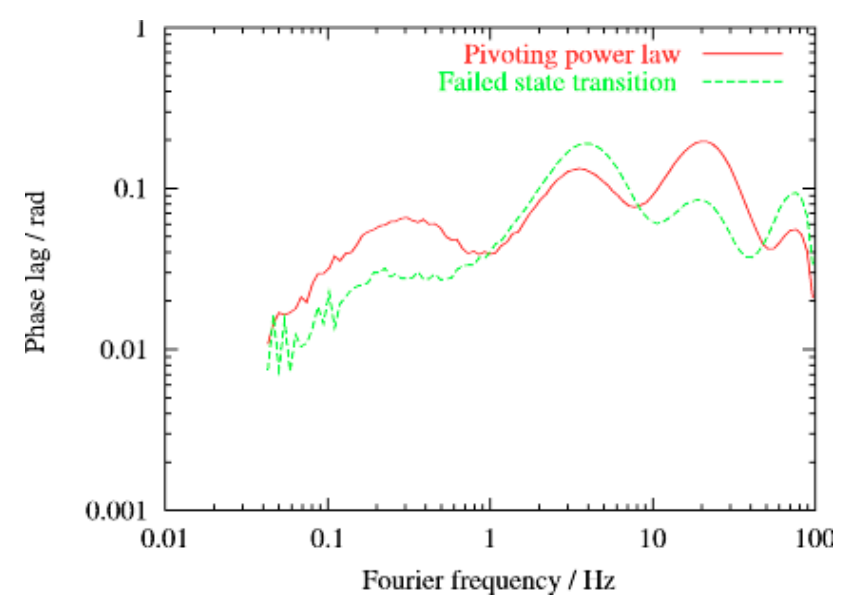

Fig. 11. Comparison of the phase lags from a normal set of Lorentzians and a "failed state transition", where the Lorentzian two dominates.

unchanged. This leads to an increase of the phase lags, where the second Lorentzian dominates, but decreases the lags further away. The increase and decrease of the phase lags depend on the other model parameters as well. While increasing lags in the $3-10 \mathrm{~Hz}$ regime are observed (Pottschmidt et al. 2003), the decrease can not be found in their plot. However, during state transitions other model parameters may change. It may be that the look-back time $\tau$ or the hardness flux correlation increases, resulting in larger lags. To fully understand this intriguing phenomenon a more detailed study is needed.

\section{Summary and conclusions}

In this paper we have discussed the effect of a variable pivoting power law in the spectrum of an astrophysical source on its timing behavior, particularly for the Fourier phase lags (see e.g., Miyamoto \& Kitamoto 1989), the cross/auto-correlation and coherence function. This model is applied to black hole $\mathrm{X}$-ray binaries. From this approach follows immediately that the Fourier phase lag dependence on photon energy is logarithmic (see also Kotov et al. 2001), which is observed in Cyg X-1. This result is independent of the choice for the response of the power law and the coherence features of the light curve.

To derive the Fourier frequency dependence of the phase lags, the coherence properties of the light curve are needed. Hints to the coherence of XRBs can be found in the PSD, which can be fitted by a few broad Lorentzians (Nowak 2000; Pottschmidt et al. 2003; Belloni et al. 2002). A Lorentzian normally arises from a damped oscillator. Therefore, we assume that the variations of the light-curve are generated by randomly excited damped oscillators, i.e. we use a simple shot noise model (see e.g., Lochner et al. 1991) to generate our light curve. The power law index was chosen to respond linearly to flux changes including a response time $(\tau)$, i.e. the power law index responds slightly after or before total intensity changes.

The analytic calculations reduce the Fourier phase lags to a simple expression, $\sin \phi \sim \gamma \sin (\omega \tau) \ln \frac{\epsilon_{2}}{\epsilon_{1}}$ for one Lorentzian, where $\gamma$ is the flux/hardness correlation parameter and $\omega$ is the Fourier frequency. This law will break down around the center 
frequency of the Lorentzian, due to an included damping of the response of the power law and stochastic variations of the lookback time $\tau$. The phase lag will therfore simply drop to zero for Fourier frequencies much higher than the center frequency of the Lorentzian.

To obtain hard lags one has to use a positive hardness/flux correlation and a positive response time (response after the change of the flux) or a negative hardness/flux correlation and a negative response time. If the power law index changes by $\Delta \Gamma \approx 0.2$ around $\Gamma=1.7$ and the look-back time is of the order of $10 \%$ of the period of the excitation we can account for the observed magnitude of the phase lags in Cyg X-1. Soft lags can be achieved by using a negative hardness/flux correlation and a positive response time - or vice versa.

The result for the phase lags of one Lorentzian is fairly independent of the exact shape of the excitations. However, the coherence properties of the light curve become more important if one superposes different Lorentzians, as needed for Cyg X-1. Using four Lorentzians we were able to reproduce the observed hard lags of Cyg X-1 (Nowak et al. 1999) using parameters of the Lorentzians within the published range. Similar hard lags are observed for BL Lacs (Zhang 2002), where the pivoting power law model may be applicable as well. The superposition of the Lorentzians is likely to play an important role in the "failed state transitions" found by Pottschmidt et al. (2000). During these events one Lorentzians normally dominates the overall PSD, the effect of the superposition is reduced resulting in larger lags.

If one does not allow for a look-back time, a pivoting power law will nevertheless create phase lags of the order of magnitude of the observed values. However, these phase lags change their sign with Fourier frequency, which is not seen in the data. However, when using a pivoting power law model as sometimes used to explain the rms behavior (see e.g., Zdziarski et al. 2003), one has to take these lags into account.

Besides the phase lags we also calculated the auto- and cross-correlation function (see e.g., Maccarone et al. 2000). They show the qualitative correct behavior seen for Cyg X-1. It is important to note, that while the result for the phase lags is fairly independent of the parameters and the form of the damping factor, the auto-correlation function can change its qualitative behavior. For example, if one uses large look-back times and large quality factors $Q$ the higher energy auto-correlation function will have a broader peak than the one for lower energy, the opposite of what observations suggest for XRBs.

The coherence function, which measures the linear correlation between the light-curves at different photon energies (Vaughan \& Nowak 1997), has been calculated and compared with observations of Cyg X-1. The model is in agreement with the observations, for Fourier frequencies where the numerical model is valid.

Both models (the jet/synchrotron model and the disk/corona models) predict the existence of a pivoting power law. At least the jet/synchrotron model creates a rigid power law without spectral breaks. We have shown that such a rigid power law model is consistent with the data of Cyg X-1. A more detailed analysis using probably an original light-curve and physical parameters for both models is needed. We also point out, that Cyg X-1 cannot be described by a simple power law (see e.g., Di Salvo et al. 2001), indicating contributions from different emission regimes that most certainly will also complicate the timing behavior.

We conclude that with a rather simple ansatz for a pivoting power law model we can explain many of the complex features in the phase lags, cross- correlation function and coherence function seen in the hard power law emission of XRBs.

Acknowledgements. The authors would like to thank an anonymous referee for very useful comments and suggestions that helped to improve the manuscript.

\section{Appendix A: Derivation of the phase lags}

The Fourier transform of a damped oscillator is a sum of two similar terms. To simplify the calculation let us first consider only the first term

$F_{1}(\omega)=\frac{2 R \sqrt{Q \omega_{0}}}{\omega_{0}-2 \mathrm{i} Q\left(\omega-\omega_{0}\right)}$.

In order to evaluate the equation for the phase lags (15) we have to calculate the convolution needed for $\widehat{\beta A}$ (Eq. (16)):

$$
\begin{aligned}
\widehat{\beta A}= & \gamma \int \mathrm{e}^{\mathrm{i} \omega^{\prime} \tau} F_{1}\left(\omega^{\prime}\right) F_{1}\left(\omega-\omega^{\prime}\right) \sum \lambda_{i} \lambda_{j} \mathrm{e}^{\mathrm{i}\left(t_{i}-t_{j}\right) \omega^{\prime}+\mathrm{i} t_{j} \omega} \mathrm{d} \omega^{\prime} \\
& +A_{\mathrm{DC}} F_{1}(\omega) \sum \lambda_{j} \mathrm{e}^{\mathrm{i} t_{j} \omega}
\end{aligned}
$$

The excitation times $t_{i}$ are random variables, therefore the sum over the non-diagonal elements will yield zero as the random phases average out.

The integral can be solved using complex analysis. It has two poles: one from $F_{1}\left(\omega^{\prime}\right)$ at $\omega^{\prime}=\omega_{0}-\mathrm{i} \frac{\omega_{0}}{2 Q}$ and the other from $F_{1}\left(\omega-\omega^{\prime}\right)$ near $\omega^{\prime}=\omega-\omega_{0}+\mathrm{i} \frac{\omega_{0}}{2 Q}$. If $\tau$ is positive, we could close the integral path with a half circle for positive imaginary parts. This path only includes the pole at $\omega^{\prime}=\omega-\omega_{0}+i \frac{\omega_{0}}{2 Q}$. Now we can apply the residual theorem, and find for the integral

$\frac{2 \pi R^{2} \omega_{0}}{\omega_{0}-\mathrm{i} Q\left(\omega-2 \omega_{0}\right)} \mathrm{e}^{-\tau \frac{\omega_{0}}{2 Q}+\mathrm{i} \tau\left(\omega-\omega_{0}\right)}$.

Inserting this expression in Eq. (15) we get

$$
\begin{aligned}
\sin \phi=\gamma \ln \frac{\epsilon_{2}}{\epsilon_{1}}\left(A_{\mathrm{DC}} \sin (\tau \omega)\right. \\
\left.+\frac{\mathfrak{J}\left(\frac{2 \pi R^{2} \omega_{0}}{\omega_{0}-\mathrm{i} Q\left(\omega-2 \omega_{0}\right)} \mathrm{e}^{-\tau \frac{\omega_{0}}{2 Q}+\mathrm{i} \tau\left(\omega-\omega_{0}\right)} F_{1}^{*}(\omega) \sum \lambda_{i}^{3}\right)}{\sum \lambda_{i}^{2} F_{1}^{*} F_{1}(\omega)}\right) .
\end{aligned}
$$

The real instantaneously excited, damped oscillator is a sum of two terms like $F_{1}(\omega)$. The result will therefore consist of four terms, which can be calculated in analogy. We find for a positive look-back time $\tau$ :

$\sin \phi=\gamma \ln \frac{\epsilon_{2}}{\epsilon_{1}}\left(A_{\mathrm{DC}} \sin (\tau \omega)+\frac{\left\langle\lambda^{3}\right\rangle}{\left\langle\lambda^{2}\right\rangle} \mathfrak{J}\left(\mathrm{e}^{\mathrm{i} \tau \omega} f_{1}(\omega)\right)\right)$, 
with

$$
\begin{aligned}
f_{1}(\omega)= & \frac{\pi R^{2} \omega_{0} F^{*}(\omega) \mathrm{e}^{-\tau \frac{\omega}{2 Q}}}{2 F^{*} F(\omega)}\left(\frac{\mathrm{e}^{-\mathrm{i} \tau \omega_{0}}}{\omega_{0}-\mathrm{i} Q\left(\omega-2 \omega_{0}\right)}\right. \\
& \left.+\frac{\mathrm{e}^{\mathrm{i} \tau \omega_{0}}}{\omega_{0}-\mathrm{i} Q\left(\omega+2 \omega_{0}\right)}+\frac{\left(\mathrm{e}^{\mathrm{i} \tau \omega_{0}}+\mathrm{e}^{-\mathrm{i} \tau \omega_{0}}\right)}{\omega_{0}-\mathrm{i} Q \omega}\right) .
\end{aligned}
$$

The other real solution for the damped oscillator is the sine term (see Eq. (9)). For this solution one has to change the two last signs and include a factor $-\mathrm{i}$. The only fast changing part of the second term of Eq. (A.5) is $\mathrm{e}^{\mathrm{i} \tau \omega}$. Therefore, the dominant part will be $\sin (\tau \omega)$ as the first term.

In the second case, $\tau<0$ we have to close the integral path in Eq. (A.2) with a half circle in the negative imaginary plane. The integral path now includes the pole at $\omega^{\prime}=\omega_{0}-\mathrm{i} \frac{\omega_{0}}{2 Q}$ and the residual theorem yields:

$\frac{2 \pi R^{2} \omega_{0}}{\omega_{0}-\mathrm{i} Q\left(\omega-2 \omega_{0}\right)} \mathrm{e}^{\tau \frac{\omega_{0}}{2 Q}+\mathrm{i} \tau \omega_{0}}$,

and we find for the phase lags:

$$
\begin{aligned}
\sin \phi=\gamma & \ln \frac{\epsilon_{2}}{\epsilon_{1}}\left(A_{\mathrm{DC}} \sin (\tau \omega)\right. \\
& \left.+\frac{\mathfrak{J}\left(\frac{2 \pi R^{2} \omega_{0}}{\omega_{0}-\mathrm{i} Q\left(\omega-2 \omega_{0}\right)} \mathrm{e}^{\tau \frac{\omega_{0}}{2 Q}+\mathrm{i} \tau \omega_{0}} F_{1}(\omega) \sum \lambda_{i}^{3}\right)}{\sum \lambda_{i}^{2} F_{1}^{*} F_{1}(\omega)}\right) .
\end{aligned}
$$

The real instantaneously excited oscillator yields for this case: $\sin \phi=\gamma \ln \frac{\epsilon_{2}}{\epsilon_{1}}\left(A_{\mathrm{DC}} \sin (\tau \omega)+\frac{\left\langle\lambda^{3}\right\rangle}{\left\langle\lambda^{2}\right\rangle} \mathfrak{J}\left(f_{2}(\omega)\right)\right.$,

with

$$
\begin{aligned}
f_{2}(\omega)= & \frac{\pi R^{2} \omega_{0} \mathrm{e}^{\tau \frac{\omega_{0}}{2 Q}}}{2 F^{*} F(\omega)}\left(\frac{\mathrm{e}^{\mathrm{i} \tau \omega_{0}} F(\omega)}{\omega_{0}-\mathrm{i} Q\left(\omega-2 \omega_{0}\right)}\right. \\
& \left.+\frac{\mathrm{e}^{-\mathrm{i} \tau \omega_{0}} F(\omega)}{\omega_{0}-\mathrm{i} Q\left(\omega+2 \omega_{0}\right)}+\frac{\left(\mathrm{e}^{-\mathrm{i} \tau \omega_{0}}+\mathrm{e}^{\mathrm{i} \tau \omega_{0}}\right) F(\omega)}{\omega_{0}-\mathrm{i} Q \omega}\right)
\end{aligned}
$$

\section{Appendix B: Derivation of the cross-correlation function}

In order to evaluate the second integral of the cross-correlation function (Eq. (21))

$$
\int\left(\hat{A}^{*} \widehat{\beta A} \ln \epsilon_{2}+\widehat{\beta A}^{*} \hat{A} \ln \epsilon_{1}\right)_{\mathrm{AC}} \mathrm{e}^{-\mathrm{i} \omega \bar{\tau}} \mathrm{d} \omega
$$

we start with Eq. (A.2) for $\widehat{\beta A}$. Using this result it is possible to evaluate the integral with the same arguments as used for $\widehat{\beta A}$. We define three supplementary functions

$$
\begin{aligned}
\mathcal{H}_{1}(\tau, \bar{\tau})= & \frac{\mathrm{e}^{-\mathrm{i} \bar{\tau} \omega_{0}}}{3 \omega_{0}+\mathrm{i} 2 Q \omega_{0}}+\frac{\mathrm{e}^{-\mathrm{i}(2 \tau-\bar{\tau}) \omega_{0}}}{3 \omega_{0}+6 \mathrm{i} Q \omega_{0}} \\
& +2 \operatorname{Cos}\left(\tau \omega_{0}\right) \frac{\mathrm{e}^{\mathrm{i}(\tau-\bar{\tau}) \omega_{0}}}{3 \omega_{0}-2 \mathrm{i} Q \omega_{0}}+\mathrm{cc} .
\end{aligned}
$$

$$
\begin{aligned}
\mathcal{H}_{2}(\tau, \bar{\tau})= & \frac{\mathrm{e}^{\mathrm{i} \tau \omega_{0}-2 \mathrm{i} \bar{\tau} \omega_{0}}}{3 \omega_{0}+2 \mathrm{i} Q \omega_{0}}+\frac{\mathrm{e}^{\mathrm{i} \tau \omega_{0}-2 \mathrm{i} \bar{\tau} \omega_{0}}}{3 \omega_{0}+6 \mathrm{i} Q \omega_{0}} \\
& +2 \operatorname{Cos}\left(\tau \omega_{0}\right) \frac{1}{3 \omega_{0}-2 \mathrm{i} Q \omega_{0}}+c c,
\end{aligned}
$$

where cc denotes the complex conjugate, and

$$
\begin{aligned}
S(\tau, \bar{\tau})= & R^{3} \omega_{0}^{3 / 2} Q^{-1 / 2} \pi^{2} \mathrm{e}^{-\tau \frac{\omega_{0}}{2 Q}}\left(\Theta_{\tau-\bar{\tau}} \mathrm{e}^{-(\tau-\bar{\tau}) \frac{\omega_{0}}{2 Q}} \mathcal{H}_{1}(\tau, \bar{\tau})\right. \\
& \left.+\Theta_{\bar{\tau}-\tau} \mathrm{e}^{2(\tau-\bar{\tau}) \frac{\omega_{0}}{2 Q}} \mathcal{H}_{2}(\tau, \bar{\tau})\right) \sum \lambda^{3},
\end{aligned}
$$

where $\Theta_{x}$ is the step function. It is defined as $\Theta_{x}=0$ for $x<0$ and $\Theta_{x}=1$ otherwise. We find for the cross-correlation function:

$$
\begin{aligned}
C\left(\epsilon_{1}, \epsilon_{2}, \bar{\tau}\right)= & P(\bar{\tau})+\gamma\left(\ln \epsilon_{1} P(\bar{\tau}-\tau)+\ln \epsilon_{2} P(\bar{\tau}+\tau)\right) \\
& +\gamma\left(\ln \epsilon_{2} S(\tau, \bar{\tau})+\ln \epsilon_{1} S(\tau,-\bar{\tau})\right) .
\end{aligned}
$$

The first line of this equation will broaden the auto-correlation function $\left(\epsilon_{1}=\epsilon_{2}\right)$ for higher photon energies, positive lookback times $\tau$, and positive hardness flux correlation $\gamma>0$. However, $\mathcal{S}(\tau, \bar{\tau})$ peaks at $\bar{\tau}=\tau$ and will decline faster for higher $\tau>\bar{\tau}$ than $P(\tau)$ (falls with $\mathrm{e}^{-\bar{\tau}}$ ) due to the factor $\mathrm{e}^{2(\tau-\bar{\tau})}$. This leads to steeper auto-correlation functions for higher $\epsilon$. Which effect dominates depends on the parameters used.

For negative look-back times $\tau$ and $\gamma<0$ we have to use Eq. (A.7) for the integral of Eq. (A.2) resulting in a different cross-correlation function. The overall form will stay the same, as Eq. (A.7) differs from Eq. (A.3) only by the argument in the exponential function - the analytic behavior (poles) is the same. Here the first line of Eq. (B.5) leads to a steeper autocorrelation function $(\gamma<0)$ while the second line has the opposite effect.

\section{References}

Böttcher, M., Jackson, D. R., \& Liang, E. P. 2003, ApJ, 586, 389 Belloni, T., Psaltis, D., \& van der Klis, M. 2002, ApJ, 572, 392 Chiang, J., Reynolds, C. S., Blaes, O. M., et al. 2000, ApJ, 528, 292

Di Salvo, T., Done, C., Życki, P. T., Burderi, L., \& Robba, N. R. 2001, ApJ, 547, 1024

Falcke, H., \& Biermann, P. L. 1999, A\&A, 342, 49

Falcke, H., Körding, E., \& Markoff, S. 2003 [astro-ph/0305335]

Fanaroff, B. L., \& Riley, J. M. 1974, MNRAS, 167, 31P

Fender, R. P. 2001, MNRAS, 322, 31

Feng, Y. X., Li, T. P., \& Chen, L. 1999, ApJ, 514, 373

Ford, E. C. 1999, ApJ, 519, L73

Haardt, F., \& Maraschi, L. 1991, ApJ, 380, L51

Homan, J., Wijnands, R., van der Klis, M., et al. 2001, ApJS, 132, 377

Kotov, O., Churazov, E., \& Gilfanov, M. 2001, MNRAS, 327, 799

Kylafis, N. D., \& Klimis, G. S. 1987, ApJ, 323, 678

Lamer, G., McHardy, I. M., Uttley, P., \& Jahoda, K. 2003, MNRAS, 338,323

Li, T. P., Feng, Y. X., \& Chen, L. 1999, ApJ, 521, 789

Lochner, J. C., Swank, J. H., \& Szymkowiak, A. E. 1991, ApJ, 376, 295

Maccarone, T. J., Coppi, P. S., \& Poutanen, J. 2000, ApJ, 537, L107

Malzac, J., \& Jourdain, E. 2000, A\&A, 359, 843

Markoff, S., Falcke, H., \& Fender, R. 2001, A\&A, 372, L25

Mirabel, I. F., \& Rodríguez, L. F. 1999, ARA\&A, 37, 409 
Miyamoto, S., Kimura, K., Kitamoto, S., Dotani, T., \& Ebisawa, K. 1991, ApJ, 383, 784

Miyamoto, S., \& Kitamoto, S. 1989, Nature, 342, 773

Narayan, R., \& Yi, I. 1995, ApJ, 452, 710

Negoro, H., Kitamoto, S., \& Mineshige, S. 2001, ApJ, 554, 528

Nolan, P. L., Gruber, D. E., Matteson, J. L., et al. 1981, ApJ, 246, 494

Nowak, M. A. 2000, MNRAS, 318, 361

Nowak, M. A., Vaughan, B. A., Wilms, J., Dove, J. B., \& Begelman, M. C. 1999, ApJ, 510, 874

Pottschmidt, K., Wilms, J., Nowak, M. A., et al. 2000, A\&A, 357, L17

Pottschmidt, K., Wilms, J., Nowak, M. A., et al. 2003, A\&A, 407, 1039

Poutanen, J., \& Fabian, A.-C. 1999, MNRAS, 306, L31

Poutanen, J. 2002, MNRAS, 332, 257

Psaltis, D., \& Norman, C. 2002 [astro-ph/0001391]

Shapiro, S. L., Lightman, A. P., \& Eardley, D. M. 1976, ApJ, 204, 187
Spencer, R. E. 1979, Nature, 282, 483

Spruit, H. C., \& Kanbach, G. 2002, A\&A, 391, 225

Sunyaev, R. A., \& Trümper, J. 1979, Nature, 279, 506

Terrell, N. J. J. 1972, ApJ, 174, L35

Urry, C. M., \& Padovani, P. 1995, PASP, 107, 803

van der Klis, M. 1989, in Timing Neutron Stars, ed. H. Ögelman, \& E. P. J. van den Heuvel (Dordrecht: Kluwer), 27

van der Klis, M. 1994, ApJS, 92, 511

van der Klis, M. 1995, in X-ray Binaries, ed. W. H. G. Lewin,

J. van Paradijs, \& E. P. J. van den Heuvel (Cambridge: Univ.

Cambridge Press), 252

Vaughan, B. A., \& Nowak, M. A. 1997, ApJ, 474, L43

Zdziarski, A. A., Lubiński, P., Gilfanov, M., \& Revnivtsev, M. 2003, MNRAS, 342, 355

Zhang, Y. H. 2002, MNRAS, 337, 609 